\title{
EDITORIAL
}

\section{Rho kinase inhibitors for pulmonary hypertension: waiting for clinical evidence}

\author{
P. Pacaud and G. Loirand
}

$\mathbf{P}$ ulmonary arterial hypertension $(\mathrm{PAH})$ is a rare disease characterised by a progressive increase of pulmonary vascular resistance (PVR) and pulmonary arterial pressure $(P \mathrm{pa})$, leading to right ventricle hypertrophy and death $[1,2]$. Although major advances in the understanding of disease development and treatment have been achieved over the past two decades, the pathogenesis of PAH is still not clearly understood. Idiopathic, familial and connective tissue disease-associated forms of PAH display similar pathological changes and are considered to share common pathogenic mechanisms, which involve endothelial dysfunction, endothelial and smooth muscle cell proliferation, increased vasoconstriction and in situ thrombosis [2]. The consequence of this process is right ventricular ischaemia, remodelling, dilation, dysfunction and failure [3]. Accordingly, current therapies are based on the use of drugs that improve endothelial function. Pharmacological agents targeting the endothelin (ET)-1 (ET-1 receptor antagonists, such as bosentan or sitaxentan), the nitric oxide (sildenafil, type 5 phosphodiesterase inhibitor), or the prostacyclin (epoprostenol, iloprost) pathways have shown benefits for patients with PAH [4]. However, these treatments failed to improve the long-term survival and their use is hampered by either side-effects or inconvenient drug administration routes [5]. Indeed, none of the currently available therapies is curative, so the search for new targets for novel therapeutic strategies continues.

Among promising targets recently identified is the serinethreonine kinase Rho kinase. Two isoforms of Rho kinase, Rock-1 and Rock-2, have been identified and found to be expressed in arteries. Rho kinase regulates a variety of cellular functions including motility, proliferation, apoptosis, contraction and gene expression [6]. Rho kinase is considered to be a major determinant of arterial tone, through its essential role in the regulation of the $\mathrm{Ca}^{2+}$ sensitivity of smooth muscle cell contraction. Furthermore, Rho kinase negatively controls endothelial nitric oxide synthase expression and activity, and participates in platelet aggregation [6]. Recent pharmacological studies suggest that activation of the small $G$ protein RhoA and its target Rho kinase is a critical shared mechanism in the pathogenesis of PAH. In vivo, potent effects of treatment with Rho kinase inhibitor (Y-27632 or fasudil) have been demonstrated in several animal models of PAH [7-10]. In addition,

INSERM, UMR915, and University of Nantes, L'institut du thorax, Nantes, France.

CORRESPONDENCE: P. Pacaud, Inserm UMR915, IRT-UN, 8 Quai Moncousu, BP 70721, 44007 Nantes cedex 1, France. E-mail: pierre.pacaud@univ-nantes.fr the beneficial effect of sildenafil on PAH is mediated, at least in part, by the inhibition of the RhoA/Rho kinase pathway [11]. In patients with $\mathrm{PAH}$, acute intravenous administration of lowdose fasudil reduced PVR and $P \mathrm{pa}[12,13]$. Acute vasodilator effects of inhaled fasudil has also been described in patients with PAH [14]. Recently, measurements of RhoA and Rho kinase activity provided direct evidence for RhoA and Rho kinase activation in platelets, pulmonary arteries and lungs from patients with PAH [15].

In the current issue of the European Respiratory Journal, MOUCHAERS et al. [16] examined the effect of fasudil on monocrotaline (MCT)-induced PAH in rats, in comparison with the action of bosentan and sildenafil. In this study, the authors specifically chose to analyse the effects of these three pharmacological compounds on an already developed $\mathrm{PAH}$ instead of a prevention protocol, as generally performed. It is the first time that the effect of Rho kinase inhibition, assessed in a curative protocol, is compared with those of two of the most commonly used therapies in PAH. For this, after 14 days after MCT injection, rats received a 14-day oral treatment with bosentan, sildenafil or fasudil. While all treatments reduced PVR, the strongest effect on PVR was obtained with fasudil treatment and only fasudil significantly lowered right ventricular systolic pressure (RVSP) and mean P pa. Similarly, among the three treatments, only fasudil was able to reduce the remodelling of the pulmonary arterial wall in MCT-treated rats. MouchaERs et al. [16] thus confirm in a curative treatment the beneficial effect of Rho kinase inhibition in PAH, and suggest a better efficiency of fasudil than bosentan or sildenafil.

Although PAH is characterised by elevated $P$ pa, the level of $P$ pa has only modest prognostic significance [17]. Rather, it is the ability of the right ventricle to function under this increased load that determines both the severity of symptoms and survival [18]. Pressure overload induces right ventricle hypertrophy that can be considered, at first, as a beneficial adaptative response. However, sustained pressure overload may lead to dilatation, fibrosis and dysfunction of the right ventricle and, eventually, right-sided heart failure [19]. Right ventricle function has a major impact on morbidity and mortality in patients with a pressure overloaded right side of the heart. Accordingly, irrespective of the aetiology of the PAH, most patients die from intractable right heart failure. However, the mechanisms involved in the development of right ventricle hypertrophy, remodelling and failing of the right ventricle and the potential of pharmacological intervention on these 
processes are poorly understood. An understanding of right ventricle hypertrophy and failure signalling will allow for future therapies to produce a stable molecular and cellular response to adverse haemodynamic stress. Conversely, pharmacological compounds that disrupt intracellular signalling involved in the response would lead to maladaptive remodelling, decompensation and right ventricle failure. MOUCHAERS et al. [16] thus specifically addressed the effect of fasudil on right ventricle function and hypertrophy in MCT-treated rats. Stroke volume and cardiac output that were both decreased in MCTtreated rats were restored by all three treatments. All treatments also reduced right ventricle dilatation. According to tricuspid annular plane systolic excursion, used as an index of right ventricle contractility, all treatments significantly improved right ventricle contractility. Assessment of right ventricle hypertrophy by the calculation of the ratio between the right ventricle and the left ventricle plus septum, and the measurements of the cross-sectional area of right ventricle cardiomyocytes, indicated that among the three treatments, only fasudil reduced right ventricle hypertrophy, in agreement with its marked effect on RVSP and mean P pa. This thus suggests that the anti-proliferative effects of Rho kinase inhibition in PAH are limited to the lung vasculature, without affecting right ventricle function and right ventricle adaptation to $\mathrm{PAH}$.

The pathogenesis of PAH is underlain by multiple cellular and molecular processes that provide the theoretical basis for the implementation of combination therapy. The goal of combination therapy is to add individual therapeutic actions to maximise efficacy, while minimising toxicity. The safety and efficacy of combination therapy in $\mathrm{PAH}$ is a subject of active investigation. Several small, open-label observational studies have suggested benefit of combination therapy using drugs with different mechanisms of action [5, 20, 21]. However, longterm, adequately powered, prospective, randomised, doubleblind, placebo-controlled studies are absolutely needed to conclusively determine the effect of combination therapy in $\mathrm{PAH}$. At the same time, animal models of PAH were also found to be useful for the assessment of the benefits of combination therapy. In MCT-treated rats, in a prevention protocol, the combination therapy of fasudil and prostacyclin, when compared with each monotherapy, showed significantly more improvement in $\mathrm{PAH}$, right ventricle hypertrophy and pulmonary medial thickness without any adverse effects [22]. In the study by MOUCHAERs et al. [16], the relevance of the combination of fasudil with bosentan and sildenafil has also been examined. Using their curative protocol in MCT-treated rats, the authors were not able to detect synergistic effect of bosentan or sildenafil in combination with fasudil on cardiac function, haemodynamics, pulmonary artery remodelling and right ventricle hypertrophy. This finding further confirms the role of Rho kinase as a convergent point in the signalling pathways involved in the pathogenesis of PAH and supports the involvement of an inhibitory action on Rho kinase pathway in the beneficial effect of sildenafil in PAH [11]. Such inhibitory action on Rho kinase activity is probably also produced by bosentan treatment as ET-1 activates Rho kinase [23]. These observations also suggest that combination therapies are relevant and expected to be efficient only if each drug used affects different and independent pathways, thus allowing synergisms or addition of each individual action.
While the study from MOUCHAERs et al. [16] constitutes a new contribution to the recognition of Rho kinase inhibitors as promising therapeutic agents through the use of an animal model of PAH, only clinical studies would now confirm the interest and the efficiency of these compounds. Although acute intravenous administration or inhalation of fasudil reduced PVR and Ppa in patients with PAH [12-14], long-term fasudil administration in patients with severe $\mathrm{PAH}$ is now absolutely required before more definitive conclusions can be made regarding the efficacy and the safety of this kind of agent. Clinical trials suggested that oral fasudil is well tolerated without any serious adverse reactions. A multicentre phase II study in patients with stable effort angina pectoris showed that a 4-week oral treatment with fasudil significantly prolongs the maximum exercise time without any effect on blood pressure and heart rate during exercise [24]. Results from large clinical studies in patients with PAH are now expected to conclude on the effectiveness of fasudil, in comparison with common therapies and with a special focus on right ventricle function.

\section{STATEMENT OF INTEREST}

None declared.

\section{REFERENCES}

1 Rubin LJ. Pulmonary arterial hypertension. Proc Am Thorac Soc 2006; 3: 111-115.

2 Humbert M. Update in pulmonary arterial hypertension 2007. Am J Respir Crit Care Med 2008; 177: 574-579.

3 McLaughlin VV, Archer SL, Badesch DB, et al. ACCF/AHA 2009 expert consensus document on pulmonary hypertension: a report of the American College of Cardiology Foundation Task Force on Expert Consensus Documents and the American Heart Association: developed in collaboration with the American College of Chest Physicians, American Thoracic Society, Inc., and the Pulmonary Hypertension Association. Circulation 2009; 119: 2250-2294.

4 Galie N, Hoeper MM, Humbert M, et al. Guidelines for the diagnosis and treatment of pulmonary hypertension. Eur Respir J 2009; 34: 1219-1263.

5 Humbert M, Sitbon O, Simonneau G. Treatment of pulmonary arterial hypertension. N Engl J Med 2004; 351: 1425-1436.

6 Loirand G, Guerin P, Pacaud P. Rho kinases in cardiovascular physiology and pathophysiology. Circ Res 2006; 98: 322-334.

7 Fagan KA, Oka M, Bauer NR, et al. Attenuation of acute hypoxic pulmonary vasoconstriction and hypoxic pulmonary hypertension in mice by inhibition of Rho-kinase. Am J Physiol Lung Cell Mol Physiol 2004; 287: L656-L664.

8 Abe K, Shimokawa H, Morikawa K, et al. Long-term treatment with a Rho-kinase inhibitor improves monocrotaline-induced fatal pulmonary hypertension in rats. Circ Res 2004; 94: 385-393.

9 Nagaoka T, Morio Y, Casanova N, et al. Rho/Rho kinase signaling mediates increased basal pulmonary vascular tone in chronically hypoxic rats. Am J Physiol Lung Cell Mol Physiol 2004; 287: L665-L672.

10 Nagaoka T, Fagan KA, Gebb SA, et al. Inhaled Rho kinase inhibitors are potent and selective vasodilators in rat pulmonary hypertension. Am J Respir Crit Care Med 2005; 171: 494-499.

11 Guilluy C, Sauzeau V, Rolli-Derkinderen M, et al. Inhibition of RhoA/Rho kinase pathway is involved in the beneficial effect of sildenafil on pulmonary hypertension. Br J Pharmacol 2005; 146: 1010-1018.

12 Fukumoto $\mathrm{Y}$, Matoba $\mathrm{T}$, Ito A, et al. Acute vasodilator effects of a Rho-kinase inhibitor, fasudil, in patients with severe pulmonary hypertension. Heart 2005; 91: 391-392. 
13 Ishikura K, Yamada N, Ito M, et al. Beneficial acute effects of rhokinase inhibitor in patients with pulmonary arterial hypertension. Circ J 2006; 70: 174-178.

14 Fujita H, Fukumoto Y, Saji K, et al. Acute vasodilator effects of inhaled fasudil, a specific Rho-kinase inhibitor, in patients with pulmonary arterial hypertension. Heart Vessels 2010; 25: 144-149.

15 Guilluy C, Eddahibi S, Agard C, et al. RhoA and Rho kinase activation in human pulmonary hypertension: role of 5-HT signaling. Am J Respir Crit Care Med 2009; 179: 1151-1158.

16 Mouchaers KTB, Schalij I, de Boer MA, et al. Fasudil reduces monocrotaline-induced pulmonary arterial hypertension: comparison with bosentan and sildenafil. Eur Respir J 2010; 36: 800-807.

17 Gaine SP, Rubin LJ. Primary pulmonary hypertension. Lancet 1998; 352: 719-725.

18 Chin KM, Kim NH, Rubin LJ. The right ventricle in pulmonary hypertension. Coron Artery Dis 2005; 16: 13-18.

19 Heineke J, Molkentin JD. Regulation of cardiac hypertrophy by intracellular signalling pathways. Nat Rev Mol Cell Biol 2006; 7: 589-600.
20 Humbert M, Barst RJ, Robbins IM, et al. Combination of bosentan with epoprostenol in pulmonary arterial hypertension: BREATHE-2. Eur Respir J 2004; 24: 353-359.

21 McLaughlin VV, Oudiz RJ, Frost A, et al. Randomized study of adding inhaled iloprost to existing bosentan in pulmonary arterial hypertension. Am J Respir Crit Care Med 2006; 174: 1257-1263.

22 Tawara S, Fukumoto Y, Shimokawa H. Effects of combined therapy with a Rho-kinase inhibitor and prostacyclin on monocrotalineinduced pulmonary hypertension in rats. J Cardiovasc Pharmacol 2007; 50: 195-200.

23 Weigand L, Sylvester JT, Shimoda LA. Mechanisms of endothelin1-induced contraction in pulmonary arteries from chronically hypoxic rats. Am J Physiol Lung Cell Mol Physiol 2006; 290: L284-L290.

24 Shimokawa $\mathrm{H}$, Hiramori $\mathrm{K}$, Iinuma $\mathrm{H}$, et al. Anti-anginal effect of fasudil, a Rho-kinase inhibitor, in patients with stable effort angina: a multicenter study. J Cardiovasc Pharmacol 2002; 40: $751-761$. 